MATHEMATICS OF COMPUTATION

Volume 68, Number 225, January 1999, Pages 395-401

S $0025-5718(99) 01024-8$

\title{
GAPS BETWEEN INTEGERS WITH THE SAME PRIME FACTORS
}

\author{
TODD COCHRANE AND ROBERT E. DRESSLER
}

\begin{abstract}
We give numerical and theoretical evidence in support of the conjecture of Dressler that between any two positive integers having the same prime factors there is a prime. In particular, it is shown that the abc conjecture implies that the gap between two consecutive such numbers $a<c$ is $\gg a^{1 / 2-\epsilon}$, and it is shown that this lower bound is best possible. Dressler's conjecture is verified for values of $a$ and $c$ up to $7 \cdot 10^{13}$.
\end{abstract}

\section{INTRODUCTION}

We start with the following conjecture of Dressler.

Conjecture 1. Between any two positive integers having the same prime factors there is a prime.

If the two integers have just one prime factor then the conjecture is a trivial consequence of Bertrand's Postulate. On the other hand, the validity of the conjecture for numbers composed of 2's and 3's implies Bertrand's Postulate. Indeed, for $n \geq 5$ one can always find positive integers $i$ and $j$ such that $n \leq 2^{i} 3,2^{j} 3^{2}<2 n$. The primary reason for believing the conjecture is evidence, both numerical and theoretical, indicating that the gap between two integers with the same prime factors is relatively large.

Conjecture 2. For any $\epsilon>0$ there exists a constant $C(\epsilon)$ such that if $a<c$ are positive integers having the same prime factors, then

$$
c-a \geq C(\epsilon) a^{\frac{1}{2}-\epsilon} .
$$

It is clear that Conjecture 1 is an easy consequence of Conjecture 2 modulo good information on $C(\epsilon)$ and on the maximal gap between consecutive primes. In this paper we shall prove that Conjecture 2 in turn is an easy consequence of the abc conjecture.

Theorem 1. The abc conjecture implies Conjecture 2.

We shall also deduce the following unconditional result as a consequence of a weaker version of the abc conjecture due to Stewart and Yu [9].

Received by the editor February 24, 1996 and, in revised form, October 7, 1996.

1991 Mathematics Subject Classification. Primary 11N25, 11 N05.

Key words and phrases. Primes, abc.

The authors wish to thank the referee for his/her helpful comments, which inspired the addition of Theorem 2 and the Example to the paper.

(C)1999 American Mathematical Society 
Theorem 2. If $a<c$ are positive integers having the same prime factors, then

$$
c-a \geq C(\epsilon)(\log c)^{\frac{3}{4}-\epsilon} .
$$

If the prime factors of $a$ and $c$ are restricted to a fixed finite set $\mathcal{S}$ of primes, then we have the much stronger lower bound of Tijdeman [10],

$$
c-a>\frac{a}{(\log a)^{C}},
$$

with the drawback being that the constant $C$ depends on the set $\mathcal{S}$.

Cramér [4] conjectured that the gap between consecutive primes $p_{n}$ and $p_{n+1}$ is $O\left(\log ^{2} p_{n}\right)$, in fact he made the stronger conjecture that $\lim \sup _{n \rightarrow \infty}\left(p_{n+1}-p_{n}\right) / \log ^{2}\left(p_{n}\right)=1$. Computer searches have shown that $p_{n+1}-p_{n}<\log ^{2} p_{n}$ for values of $p_{n}$ up to $7 \times 10^{13}$; see Shanks [8], Lander and Parkin [6], Brent [1], and Young and Potler [12]. On the assumption of the Riemann Hypothesis, Cramér proved that there always exists a prime between $n$ and $n+O\left(n^{\frac{1}{2}} \log n\right)$. In order to deduce Conjecture 1 from Conjecture 2 one needs gaps of size $O\left(n^{\frac{1}{2}-\epsilon}\right)$, which is somewhere between what one obtains from the Riemann Hypothesis and what Cramér has conjectured. On the other hand, with just a "modest" impovement in Theorem 2, specifically obtaining $c-a \geq(\log c)^{2}$, Conjecture 1 is essentially a consequence of Cramér's conjecture.

The following example shows that the exponent in (1) cannot be taken to be equal to $1 / 2$. Indeed, we obtain an infinite family of pairs of positive integers $a<c$ having the same prime factors and satisfying

$$
c-a \leq \frac{2 \sqrt{2 \log 2} a^{1 / 2}}{(\log a)^{\frac{1}{2}}} .
$$

Example. Let $k$ be any positive integer and define $a_{1}, c_{1}$ by

$$
a_{1}=2\left(2^{k}-1\right)^{2}, \quad c_{1}=2^{k+1}\left(2^{k}-1\right) .
$$

Then $c_{1}, a_{1}$ have the same prime divisors and $c_{1}-a_{1}=\sqrt{2} a_{1}{ }^{1 / 2}$. Suppose now that $k=2 \cdot 3^{j-1}$, where $j \geq 2$ is a positive integer. Then we have $3^{j} \mid\left(2^{k}-1\right)$ and so we can divide $a_{1}$ and $c_{1}$ by $3^{j-1}$ and end up with two smaller numbers

$$
a=\frac{2\left(2^{k}-1\right)^{2}}{3^{j-1}}, \quad c=\frac{2^{k+1}\left(2^{k}-1\right)}{3^{j-1}}
$$

having the same prime factors and satisfying

Now,

$$
c-a=\frac{\sqrt{2}}{3^{(j-1) / 2}} a^{1 / 2}=\frac{2}{\sqrt{k}} a^{1 / 2} .
$$

$$
\log a=\log 2+2 \log \left(2^{k}-1\right)-(j-1) \log 3<2 k \log 2,
$$

that is, $k>\log a /(2 \log 2)$, and thus we obtain (2). Similar examples may be obtained by dividing out other prime powers or by replacing $\left(2^{k}-1\right)$ with $\left(2^{k}+1\right)$ or by replacing 2 with any other positive integer $m>1$, but we know of no example where the order of magnitude is less than what we obtain in (2).

If $a$ and $c$ have just two prime divisors then we show that the exponent in (1) can be taken to be equal to $1 / 2$ on the assumption of the abc conjecture.

Theorem 3. Suppose that $a<c$ are positive integers having the same two prime divisors. Then, on the assumption of the abc conjecture, $c-a \gg a^{1 / 2}$. 
In Section 3 we use results of de Weger [11] to prove (Theorem 4 in this paper) that the only positive integers $a<c$ composed of the same two primes $p, q$ with $p<q<200$ and

$$
c-a<\sqrt{a}
$$

are $(a, c)=(48,54)=\left(2^{4} \cdot 3,2 \cdot 3^{3}\right),(a, c)=(1250,1280)=\left(2 \cdot 5^{4}, 2^{8} \cdot 5\right)$ and $(a, c)=(11859482,11862016)=\left(2 \cdot 181^{3}, 2^{16} \cdot 181\right)$. The following is an open question.

Question 1. Are there infinitely many pairs $a<c$ having the same two prime factors satisfying (3)?

Using the table of Young and Potler [12] on first occurrences of prime gaps, we have been able to verify with a computer search that Conjecture 1 is valid for $a<c<7 \cdot 10^{13}$. The only example in this range with $c-a$ less than the maximal gap between primes up to $c$ is $(a, c)=(2400,2430)$. The largest gap between consecutive primes up to $7 \cdot 10^{13}$ is just 778 , substantially smaller than the cube root of $7 \cdot 10^{13}$. Thus for $n>7 \cdot 10^{13}$ it is reasonable to believe that there is always a prime between $n$ and $n+n^{1 / 3}$. In this case, Conjecture 1 follows if one can establish that for any $a<c$ having the same prime factors,

$$
c-a>a^{1 / 3} \text {. }
$$

We know of no example for which (4) fails, and so we ask

Question 2. Is there any pair $a<c$ composed of the same prime factors with $c-a<a^{1 / 3}$ ?

From de Weger's work in [11] we can obtain (Theorem 5) all solutions of (3) with $a$ and $c$ composed of the primes $2,3,5,7,11$ and 13 , and having the same prime factors. All of these solutions satisfy (4) as well. Thus (4) holds for all $a, c$ composed of the same primes from the set 2,3,5,7,11, and 13. Further examples satisfying (3) may be gleaned from the tables of Nitaj [7] and Browkin and Brzezinski [2] on extremal examples for the abc conjecture. All of these examples satisfy (4) as well.

\section{Proofs of Theorems 1 And 2}

For any positive integer $n$ let $N_{0}(n)=\Pi_{p \mid n} p$, the product being over the distinct prime factors of $n$.

The abc conjecture. For any $\epsilon>0$ there exists a constant $C(\epsilon)$ such that for any nonzero relatively prime integers $a, b$ and $c$ with $a+b=c$ we have

$$
\max (|a|,|b|,|c|) \leq C(\epsilon) N_{0}(a b c)^{1+\epsilon} .
$$

Suppose now that $a<c$ are positive integers having the same prime factors. Let $b=c-a$. Put $P=N_{0}(a)=N_{0}(c)$ and $d=(a, b)=(a, c)=(b, c)$. Then $\frac{a}{d}+\frac{b}{d}=\frac{c}{d}$ and the integers $\frac{a}{d}, \frac{b}{d}$ and $\frac{c}{d}$ are relatively prime. Now

$$
N_{0}\left(\frac{a}{d} \frac{b}{d} \frac{c}{d}\right) \leq N_{0}(a c) N_{0}\left(\frac{b}{d}\right) \leq P \frac{b}{d} \leq \frac{b^{2}}{d}
$$

the last inequality following since $P \mid b$. It follows from (5) that $\frac{c}{d} \leq C(\epsilon)\left(\frac{b^{2}}{d}\right)^{1+\epsilon}$, and so $c \leq C(\epsilon) b^{2(1+\epsilon)}$, that is $b \geq C^{\prime}(\epsilon) c^{\frac{1}{2}-\epsilon}$. This establishes Theorem 1 . 
For the proof of Theorem 2 we proceed as above but instead of applying the abc conjecture we apply the following weaker, but proven, result of Stewart and Yu [9]. Under the same assumptions as in the abc conjecture above we have

$$
\max (\log |a|, \log |b|, \log |c|) \leq C(\epsilon) N_{0}(a b c)^{\frac{2}{3}+\epsilon} .
$$

In our application we obtain

$$
\log (c / d) \ll\left(b^{2} / d\right)^{\frac{2}{3}+\epsilon},
$$

from which we deduce

$$
b^{2} \gg d(\log (c / d))^{\frac{3}{2}-\epsilon} \gg(\log c)^{\frac{3}{2}-\epsilon},
$$

which completes the proof of Theorem 2. The latter inequality follows from the claim, for $2 \leq d \leq c / 2$ and $0<\epsilon<3 / 2$ we have

$$
d(\log (c / d))^{\frac{3}{2}-\epsilon} \geq \cdot 7(\log c)^{\frac{3}{2}-\epsilon}
$$

The claim follows from observing that

$$
d\left(1-\frac{\log d}{\log c}\right)^{\frac{3}{2}-\epsilon} \geq d\left(1-\frac{\log d}{\log 2 d}\right)^{3 / 2}=d\left(\frac{\log 2}{\log 2 d}\right)^{3 / 2} \geq 2\left(\frac{\log 2}{\log 4}\right)^{3 / 2}>.7
$$

\section{THE CASE OF TWO PRIME FACTORS:}

ProOF OF THEOREM 3

Suppose that $a<c$ are positive integers composed of the same two prime divisors $p, q$. Let $(a, c)=p^{e} q^{f}$ and write

$$
c=p^{e+g} q^{f}, \quad a=p^{e} q^{f+h}, \quad b=c-a=p^{e} q^{f}\left(p^{g}-q^{h}\right) .
$$

We start by observing that in this case a large gap between $a$ and $c$ is tantamount to a large gap between the prime powers $p^{g}$ and $q^{h}$. To be precise, the inequality

$$
c-a \gg a^{1 / 2}
$$

is equivalent to the inequality

$$
p^{g}-q^{h} \gg p^{\frac{g}{2}\left(1-\frac{f}{h}-\frac{e}{g}\right)} .
$$

To see this we consider two cases. If $q^{h}<\frac{1}{2} p^{g}$, then (8) and (9) are both trivially true, and so we may assume that $\frac{1}{2} p^{g} \leq q^{h}<p^{g}$. Now (8) is equivalent to

$$
p^{g}-q^{h} \gg p^{\frac{-e}{2}} q^{\frac{-f+h}{2}} \text {. }
$$

Substituting $q \approx p^{g / h}$ into the right-hand side yields (9).

We conclude the proof of Theorem 3 by showing that (9) holds true under the assumption of the abc conjecture.

It suffices to consider the case $e=f=1$ whence (9) becomes

$$
p^{g}-q^{h} \gg p^{\frac{g}{2}\left(1-\frac{1}{h}-\frac{1}{g}\right)} \text {. }
$$

If $h=1$ or $g=1$ or $(h, g)=(2,2)$, then (10) is trivial. Thus we may assume that $h \geq 2, g \geq 2$, and that either $h$ or $g$ is $\geq 3$. Now, the abc conjecture, applied to the sum $p^{g}-q^{h}=\left(p^{g}-q^{h}\right)$, implies that

$$
p^{g} \ll\left(p q\left|p^{g}-q^{h}\right|\right)^{1+\epsilon},
$$


or equivalently

$$
\left|p^{g}-q^{h}\right| \gg p^{g\left(1-\frac{1}{g}-\frac{1}{h}-\epsilon\right)},
$$

the constants depending on $\epsilon$. Since $1 / h+1 / g \leq 5 / 6$, one obtains (10) from (11) on taking $\epsilon<1 / 12$. This completes the proof of Theorem 3 .

Remark. The argument above applies just as well to any relatively prime integers $p$ and $q$ (not necessarily primes). Thus Theorem 3 is valid for any $a, c$ as in (7) with $p, q$ relatively prime positive integers.

As one can see by the equivalence of (8) and (9), finding pairs $a, c$ with $c-a$ small amounts to finding two prime powers close together. Cijsouw, Korlaar and Tijdeman [3] found all solutions of the inequality

$$
\left|p^{g}-q^{h}\right|<p^{g / 2},
$$

in positive integers $g, h$ and primes $p<q<20$. Their work was extended by de Weger ([11], Theorem 4.3) to the range $p<q<200$; see also Deze and Tijdeman ([5], Lemma 1). Now any solution of (3) with $a, b, c$ as in (7) satisfies

$$
p^{g}-q^{h}<p^{\frac{-e}{2}} q^{\frac{h-f}{2}}<p^{\frac{-1}{2}} q^{\frac{h-1}{2}} .
$$

If $p<q$, then using the fact that $q<p^{g / h}$ we obtain

$$
p^{g}-q^{h}<p^{\frac{g}{2}\left(1-\frac{1}{h}-\frac{1}{g}\right)},
$$

which is a stronger inequality than (12). If $q<p$, then $p^{-1 / 2}<q^{-1 / 2}$ and so we obtain

$$
p^{g}-q^{h}<q^{\frac{h}{2}-1},
$$

which again is stronger than (12) with the roles of $p$ and $q$ reversed. Thus all solutions of (3) with $p, q<200$ may be found by testing the solutions of (12) given by de Weger in [11]. By doing so we obtain

Theorem 4. Suppose that $a<c$ are positive integers as in (7) with $p, q<200$ and $c-a<a^{1 / 2}$. Then $(a, c)=(48,54)=\left(2^{4} \cdot 3,2 \cdot 3^{3}\right),(1250,1280)=\left(2 \cdot 5^{4}, 2^{8} \cdot 5\right)$ or $(11859482,11862016)=\left(2 \cdot 181^{3}, 2^{16} \cdot 181\right)$.

4. $a, c$ RESTRICTED TO THE PRIMES $2,3,5,7,11$ AND 13

In [11, Theorem 4.6], de Weger solved the diophantine inequality

$$
0<c-a<a^{1 / 2}
$$

with

$$
a, c \in\left\{2^{x_{1}} \ldots 13^{x_{6}}: x_{i} \in \mathbb{Z}, x_{i} \geq 0,(1 \leq i \leq 6)\right\},
$$

and $(a, c)=1$. He found exactly 605 solutions, and all of them satisfy $\nu_{2}(a c) \leq 26$, $\nu_{3}(a c) \leq 19, \nu_{5}(a c) \leq 13, \nu_{7}(a c) \leq 13, \nu_{11}(a c) \leq 7$, and $\nu_{13}(a c) \leq 8$. Here, $\nu_{p}(n)$ denotes the multiplicity of $p$ dividing $n$. We ran a program in UBASIC to test which of these satisfy the stronger inequality

$$
0<P(c-a)<(P a)^{1 / 2},
$$

where $P$ is the product of the primes appearing in $a c$. In this manner we were able to establish 
Theorem 5. There are 58 pairs of positive integers $a<c$ having the same prime factors, with the primes selected from the set $\{2,3,5,7,11,13\}$, such that $c-a<$ $a^{1 / 2}$. In every such pair we have $c<15 \cdot 10^{9}$, and $c-a>a^{1 / 3}$. Of these pairs, 19 are primitive, $(a, c)=1$.

A complete listing of the pairs in Theorem 5 is available upon request.

\section{SMALl gaPS WITh $a<c<7 \cdot 10^{13}$}

In the chart below we list all pairs $0<a<c<7 \cdot 10^{13}$, having the same prime factors, with $c-a$ less than twice the maximal gap between primes up to $c$.

$48=2^{4} \cdot 3$
$1250=2 \cdot 5^{4}$
$2016=2^{5} \cdot 3^{2} \cdot 7$
$2400=2^{5} \cdot 3 \cdot 5^{2}$
$2646=2 \cdot 3^{3} \cdot 7^{2}$
$15972=2^{2} \cdot 3 \cdot 11^{3}$
$29376=2^{6} \cdot 3^{3} \cdot 17$
$58368=2^{10} \cdot 3 \cdot 19$
$504000=2^{6} \cdot 3^{2} \cdot 5^{3} \cdot 7$
$918540=2^{2} \cdot 3^{8} \cdot 5 \cdot 7$

$\begin{array}{lll}54=2 \cdot 3^{3} & 6 & 6 \\ 1280=2^{8} \cdot 5 & 30 & 22 \\ 2058=2 \cdot 3 \cdot 7^{3} & 42 & 34 \\ 2430=2 \cdot 3^{5} \cdot 5 & 30 & 34 \\ 2688=2^{7} \cdot 3 \cdot 7 & 42 & 34 \\ 16038=2 \cdot 3^{6} \cdot 11 & 66 & 44 \\ 29478=2 \cdot 3 \cdot 17^{2} & 102 & 52 \\ 58482=2 \cdot 3^{4} \cdot 19^{2} & 114 & 72 \\ 504210=2 \cdot 3 \cdot 5 \cdot 7^{5} & 210 & 114 \\ 918750=2 \cdot 3 \cdot 5^{5} \cdot 7^{2} & 210 & 114\end{array}$

The table above was obtained by a direct search on a PC using UBASIC. The idea of the program is very simple, and it runs extremely fast. For example if $a, c$ have three odd primes in common, say $p_{1}, p_{2}, p_{3}$, then we know $p_{1} p_{2} p_{3}<778 / 2$, half the maximal gap between consecutive primes up to $7 \cdot 10^{13}$, and so the choices for $p_{1}, p_{2}$ and $p_{3}$ are very restricted, etc.

\section{REFERENCES}

[1] R. Brent, The first occurrence of certain large prime gaps, Math. Comp. 35 (1980), 14351436. MR 81g:10002

[2] J. Browkin and J. Brzezinski, Some remarks on the abc-conjecture, Math. Comp. 62 (206) (1994), 931-939. MR 94g:11021

[3] P.L. Cijsouw, A. Korlaar and R. Tijdeman, Appendix to the paper "Diophantine equations" by R.J. Stroeker and R. Tijdeman appearing in the book Computational Methods in Number Theory, Mathematical Centre Tracts 155, Mathematisch Centrum, Amsterdam, 1982, pp. 354-363. MR 84d:10004

[4] H. Cramér, On the order of magnitude of the difference between consecutive prime numbers, Acta Arith. 2 (1937), 23-46.

[5] M. Deze and R. Tijdeman, Exponential diophantine equations with four terms, Indag. Mathem., N.S. 3 (1) (1992), 47-57. MR 93d:11035

[6] L.J. Lander and T.R. Parkin, On first appearance of prime differences, Math. Comp. 35 (1980), 483-488. MR 37:6237

[7] A. Nitaj, An algorithm for finding good abc-examples, C.R. Acad. Sci. Paris 317 (1993), 811-815. MR 94k:11035

[8] D. Shanks, On maximal gaps between successive primes, Math. Comp. 18 (1964), 646-651. MR 29: 4745

[9] C.L. Stewart and K.R. Yu, On the abc conjecture, Math. Ann. 291 (1991), 225-230. MR 92k:11037 
[10] R. Tijdeman, On integers with many small prime factors, Compositio Math. 26 (3) (1973), 319-330. MR 48:3896

[11] B.M.M. de Weger, Solving exponential diophantine equations using lattice basis reduction algorithms, J. Number Th. 26 (1987), 325-367. MR 88k:11097

[12] J. Young and A. Potler, First occurence prime gaps, Math. Comp. 52 (185) (1989), 221-224. MR 89f:11019

Kansas State University, Manhattan KS 66506, U. S. A.

E-mail address: cochrane@math.ksu.edu

E-mail address: dressler@math.ksu.edu 